\title{
A Putative Relation between Obstructive Sleep Apnea and Diabetic Macular Edema Associated with Optic Nerve Fiber Layer Infarcts
}

\author{
Yaprak Banu Unver ${ }^{1}$, Gulderen S. Aktan Yavuz ${ }^{2}$, Calvin A. Stafford ${ }^{3}$ and Stephen H. Sinclair*,, \\ ${ }^{I}$ Beyoglu Eye Training and Research Hospital, Istanbul, Turkey \\ ${ }^{2}$ Duzce University Medical School, Department of Ophthalmology, Duzce, Turkey \\ ${ }^{3}$ Crozer Sleep Disorders Center, Taylor Hospital, Ridley Park, Pennsylvania, USA \\ ${ }^{4}$ Department of Ophthalmology, Drexel University College of Medicine, Philadelphia, PA, USA \\ ${ }^{5}$ Riddle Memorial Hospital, Media, PA, USA
}

\begin{abstract}
:
Purpose: To describe a possible relationship in obese diabetics between obstructive sleep apnea syndrome (OSAS) and ischemic retinopathy with diffuse macular edema.

Design: Prospective, observational case series.

Methods: Obese diabetics $(\mathrm{n}=22)$ in a retina clinic who admitted to symptoms of sleep apnea on survey were compared with a cohort of 22 similarly obese diabetic patients.

Complete ocular examination, fundus photography, fluorescein angiography,overnight polysomnography, blood pressure measurement, and blood testing for $\mathrm{HgA} 1 \mathrm{C}, \mathrm{CBC}$, lipid profile, ESR, c reactive protein, $\mathrm{BUN}$, creatinine and proteinuria were evaluated.

Results: Among the 22 obese diabetics of apnea cohort who underwent polysomnography, 16 demonstrated severe and 6 moderate OSAS. An average of $44.6 \pm 21.9$ apneic or hypopneic events per hour were recorded during sleep, lasting on average $23.3 \pm 5.9$ seconds, resulting in oxygen desaturation of $73.5 \pm 9.5 \%$. The retinopathy observed in the apnea cohort manifested multiple nerve-fiber-layer infarcts, at least 3 in each eye in all cases, and more than 6 infarcts in most eyes.. Among the cohort without sleep apnea, rare nerve fiber layer infarcts were observed, and the retinal microvascular leakage and macular edema was more focal and resolved with grid laser. Among the measured systemic factors, none showed a statistical difference between apneic and non-apneic cohorts.

Conclusions: In obese diabetics, obstructive sleep apnea appears to be associated with retinopathy of more aggressive course, manifesting multiple nerve-fiber-layer infarction and diffuse macular edema. Physicians should consider evaluating obese diabetics who have snoring, fragmented sleep patterns, daytime somnolence, hypertension or manifesting accelerated course of retinopathy similar to the patients described.
\end{abstract}

\section{INTRODUCTION}

Periods of partial or complete upper airway obstruction may occur during sleep that cause recurrent arousal and disrupt sleep quality resulting in somnolence during the day. Termed "obstructive sleep apnea syndrome" (OSAS), the airway obstruction occurs mainly in the upper pharynx [1] at the end of inspiration and predominantly but not exclusively in the supine position.

During sleep, activation of the muscles which normally maintain upper airway patency against the negative pressures of inspiration is depressed, leading to episodes of collapse of the oropharynx where there are no rigid structures. By definition, the episodes must exceed 10 seconds in duration but often last much longer, upwards of 40-75 seconds in severe instances. A minimum of 5 apnea or hypopnea episodes per hour of sleep are required to diagnose sleep apnea [1], how-

*Address correspondence to this author at the 311 Baltimore Pike, 1st Floor, 100 Suit, Media, PA 19063, USA; E-mails: Stephensinclair@mac.com, stephensinclair@comcast.net ever typical cases involve many more events, commonly several hundred in a single night or more than 20 to 30 per hour in severe cases. These episodes of breathing irregularity induce hypoxemia and hypercapnea. Oxyhemoglobin saturation frequently declines to $70-80 \%$ and levels below $60 \%$ occur occasionally (during normal sleep, oxyhemoglobin remains in excess of $94 \%$ ). The apneic episodes end with an eruptive ventilatory reprise typically associated with partial or complete arousal, at which time oxygen and carbon dioxide levels commonly return to baseline levels.

The hemodynamic consequences of the apnea events have been extensively studied. Heart rate slows during the apneic episode and accelerates briskly with the restoration of airflow [2, 3]. Mean arterial pressure may initially decline slightly but then rises slowly during the event. A marked surge in mean systemic pressure occurs after the airflow returns, sometimes rising 10-30 $\mathrm{mmHg}[4,5]$. Over the course of the night, there is commonly a progressive rise in mean arterial pressure in patients with sleep apnea [4], in marked contrast to a normal fall in blood pressure through the night. 
Sleep apnea is a documented independent risk factor for systemic arterial hypertension [5]. It contributes to pulmonary hypertension [6,7] and has been reported to be a significant risk factor for nocturnal stroke and myocardial infarction [8, 9] as well as renal failure in diabetic patients [10,11].

A major risk factor for obstructive sleep apnea is obesity. Sleep apnea is present in over $60 \%$ of patients mildly obese (body mass index of $28 \mathrm{Kg} / \mathrm{SqM}$ or approximately 212 pounds at 6 feet tall) rising to more than $80 \%$ in the very obese [12]. Deposition of fat in the tissue surrounding the pharyngeal airway narrows the aperture promoting a greater tendency for obstruction [13]. Additionally, obese individuals often have a reduced lung volume and hypoventilation frequently occur even without obstruction during sleep [14]. Obesity is likewise a major risk factor for diabetes mellitus. Over the last decade in the United States, the incidence of type II diabetes has increased $44 \%$, a rise attributed in large measure to increased prevalence of morbid obesity [15].

In the eye, obstructive sleep apnea may be a significant aggravating factor for the progression of diabetic retinal microangiopathy because of the associated hypoxemia, hypercapnia, and hypertension. After the onset of retinal diabetic microangiopathy, microvascular occlusion [16] and reduced auto-regulation [17-19] may make the retina susceptible to ischemic injury from the hypoxemia associated with sleep apnea. Hypertension, as well, has been demonstrated to be associated with an aggravated course of diabetic retinopathy primarily associated with increased frequency of nerve-fiberlayer infarcts but also with worsened macular edema [20-22].

In this paper, we present a series of obese diabetics who were documented to have moderate or severe obstructive sleep apnea and who presented with retinopathy that appeared to be unusual in terms of rapid progression of the ischemia, demonstrating multiple nerve fiber layer infarcts, and in the presence of diffuse macular edema that was extremely resistant to treatment. This cohort is compared with a historical control cohort of obese diabetics who on survey did not have sufficient symptoms to suggest obstructive sleep apnea.

\section{DESIGN}

A cohort of consecutive, obese, diabetic patients obtained from a retina referral practice who responded to a survey with significant symptoms suggesting obstructive sleep apnea was compared with a cohort of patients, matched for age, weight, and duration of diabetes, that were selected retrospectively from records but who, in the same survey, indicated minimal symptoms of obstructive sleep apnea.

\section{METHODS}

Among obese diabetic patients followed in a retina referral practice (Crozer Chester Medical Center Upland, PA) for retinopathy, 22 sequential patients were identified when they answered to survey questioning that they experienced frequent nocturnal awakening, daytime somnolence (Epworth score greater than 10 on survey, see Table 4) and when their bed partners admitted they frequently snored. Thus,the diagnosis of obstructive sleep apnea was made indirectly based on a survey questionnaire. All appeared to have a normal face, with no significant mandible recession (retrognathia) [23], but all were characterized as having a short, thick neck and with the majority also having kyphosis. This cohort was

Table 1. Characteristics and Systemic Diseases of Patients Studied

\begin{tabular}{|c|c|c|c|c|}
\hline & \multicolumn{2}{|c|}{ Patients with Obstructive Sleep Apnea } & \multicolumn{2}{|c|}{ Patients without Obstructive Sleep Apnea } \\
\hline & Mean & Number of Patients & Mean & Number of Patients \\
\hline Age (years) & $57 \pm 12.7$ & 22 & $62.1 \pm 10.8$ & 22 \\
\hline Duration of Diabetes (years) & $16.8 \pm 11.4$ & 22 & $15.7 \pm 9.4$ & 22 \\
\hline Duration IDDM & $11.9 \pm 9.3$ & 15 & $10.3 \pm 8.5$ & 13 \\
\hline Duration Hypertension (years) & $11.3 \pm 8.9$ & 19 & $13.1 \pm 6.5$ & 19 \\
\hline Current Systolic BP & $148.2 \pm 22.8$ & 22 & $144.2 \pm 26.1$ & 22 \\
\hline Current Diastolic BP & $80.4 \pm 12.7$ & 22 & $82.5 \pm 18.2$ & 22 \\
\hline \multicolumn{5}{|l|}{ Other Systemic Diseases } \\
\hline Cardiac MI or failure & & 6 & & 9 \\
\hline Renal failure & & 2 & & 1 \\
\hline Asthma & & 1 & & 0 \\
\hline CVA & & 1 & & 2 \\
\hline Other & & 1 (ITP) & & 0 \\
\hline
\end{tabular}


compared with a cohort of 22 diabetic patients selected by retrospective chart review of the same practice, and who were matched for age (within 10 years), for body mass index (within 30 pounds), for duration of diabetes (within 5 years), but who on the same somnolence survey, had responded with minimal symptoms (Epworth score less than 10). In addition to a complete ocular examination, fundus photography, and fluorescein angiography, all patients in both cohorts, in addition to measurement of blood pressure, underwent testing for HgA1C, hemoglobin, lipid profile, erythrocyte sedimentation rate, C-reactive protein, BUN, and creatinine, and 24 hour urine collection for creatinine clearance and proteinuria. Those with an Epworth score greater than 10 (indicating significant symptoms of obstructive sleep apnea) underwent overnight polysomnography. Ethical consent was received for each patient from the Crozer Chester Hospital Ethical Committee.

\section{RESULTS}

In the somnolence survey utilized to divide the diabetics into those with obstructive sleep apnea as opposed to those without, the mean Epworth score of the apnea group was 13.5 compared to 6.4 in the cohort without apnea selected for comparison. The descriptive statistics are presented in Table 1 for each cohort. The mean age for the sleep apnea group was $57 \pm 12.7( \pm 1$ S.D.) years with a mean duration of diabetes of $16.8 \pm 11.4$ yrs. For those without sleep apnea the mean age was $62.1 \pm 10.8$ years with duration of diabetes of $15.7 \pm 9.4$ years. Fifteen of those in the apnea group were insulin dependent at the time of sleep apnea diagnosis while only 13 were insulin dependent in the group without sleep apnea. Weight averaged $249 \pm 33.5$ pounds in the apnea group and $245.8 \pm 44.6$ pounds in the non-apnea group. Nineteen of the patients with apnea had a history of hypertension for an av- erage of $11.3 \pm 8.9$ years, and most were on multiple medications to control the hypertension. The average systolic blood pressure at the time of sleep apnea diagnosis was $148.2 \pm 22.8$ while the average diastolic blood pressure measured $80.4 \pm 12.7 \mathrm{mmHg}$. Among those without sleep apnea, the blood pressure measurements were similar with duration of hypertension slightly longer at $13.1 \pm 6.5$ years.

The retinopathy observed in all patients with apnea was characterized by multiple nerve-fiber-layer infarcts, at least 3 in each eye in all cases, and more than 6 infarcts observed in most eyes. The nerve fiber layer infarcts appeared in multiple stages of evolution often with new lesions observed on follow-up fundoscopy. The periphery was characterized on fluorescein angiography as having widespread capillary nonperfusion in 14 of the patients. In contrast, among those obese diabetics without apnea, rare nerve fiber layer infarcts were observed (in 5 patients) and fluorescein angiography demonstrated scattered foci of microaneurysms in the periphery sometimes with a few scattered, focal areas of capillary telangiectasia but with significantly less areas of scattered or composite capillary non-perfusion. Among the patients with sleep apnea, the macula in 20 demonstrated diffuse edema, often with focal areas of macular capillary nonperfusion. Among those without sleep apnea, macular edema was observed in 9 patients and was due to more localized areas of leakage and without posterior, macular capillary non-perfusion. Fundus photographs and fluorescein angiograms of three patients representative of those with obstructive sleep apnea are presented in Figs. (1-3). Seven of the patients at the time of sleep apnea diagnosis had undergone at least one trial of grid laser for the edema, five had undergone at least two treatments in each eye, and in two patients each eye had undergone three or more laser treatments. In those patients who had undergone grid laser photocoagula-
A

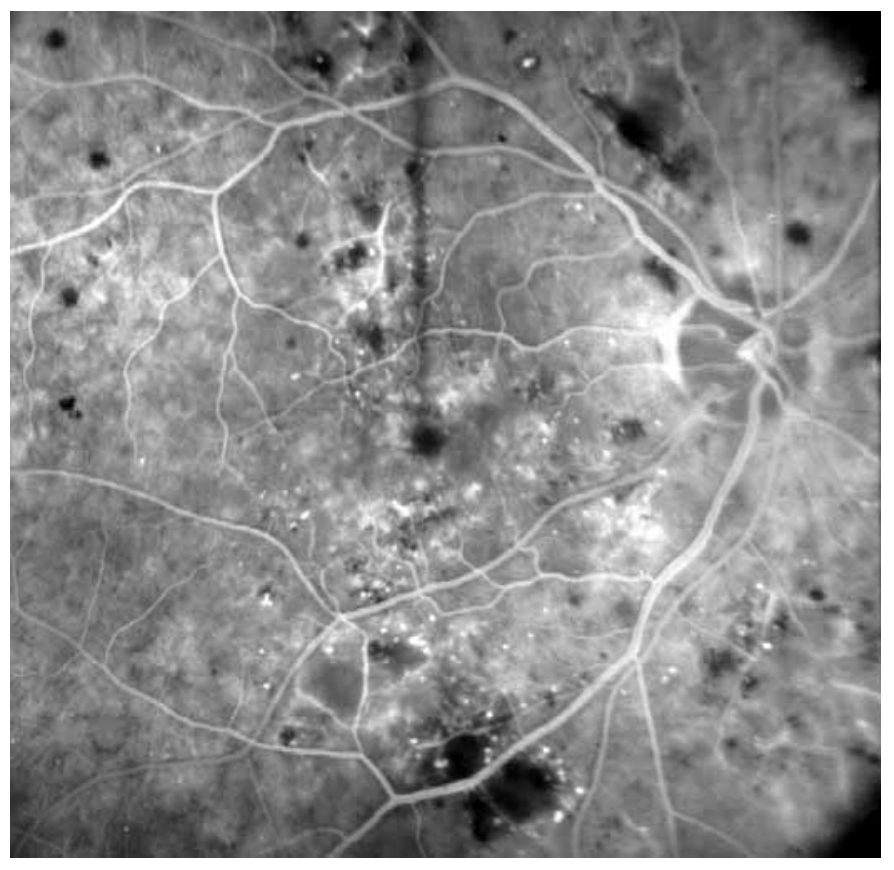

B

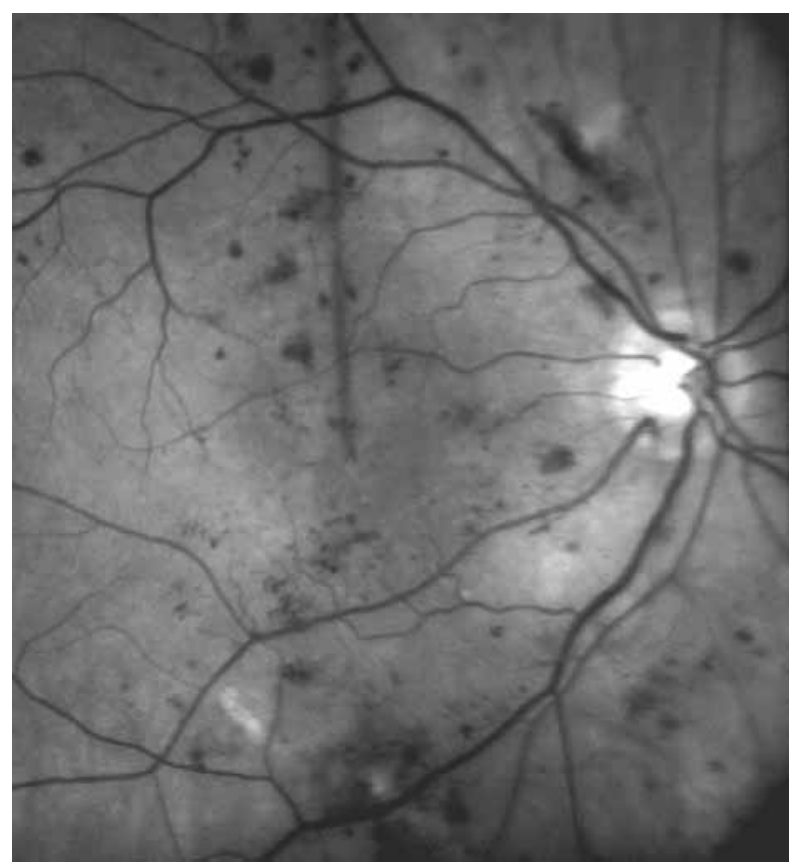

Fig. (1). Patient \#1: OD Retinal green filter photograph (A) and capillary phase fluorescein angiogram (B) demonstrating multiple nervefiber layer infarcts, dot, blot, and striate hemorrhages, and diffuse macular telangiectasia with leakage. 
A

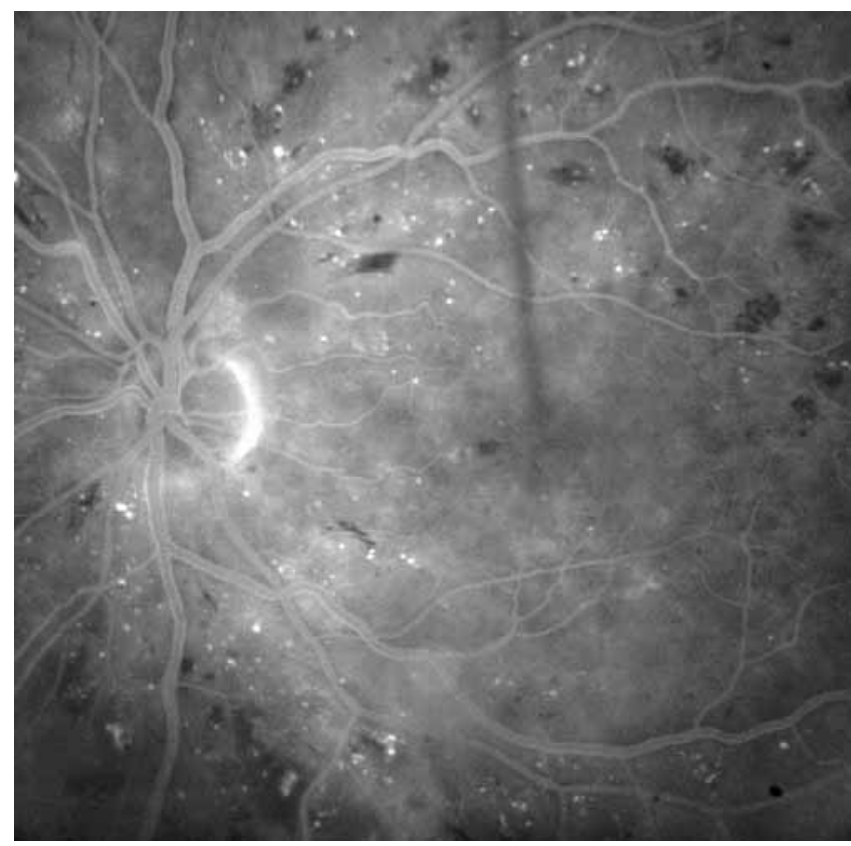

B

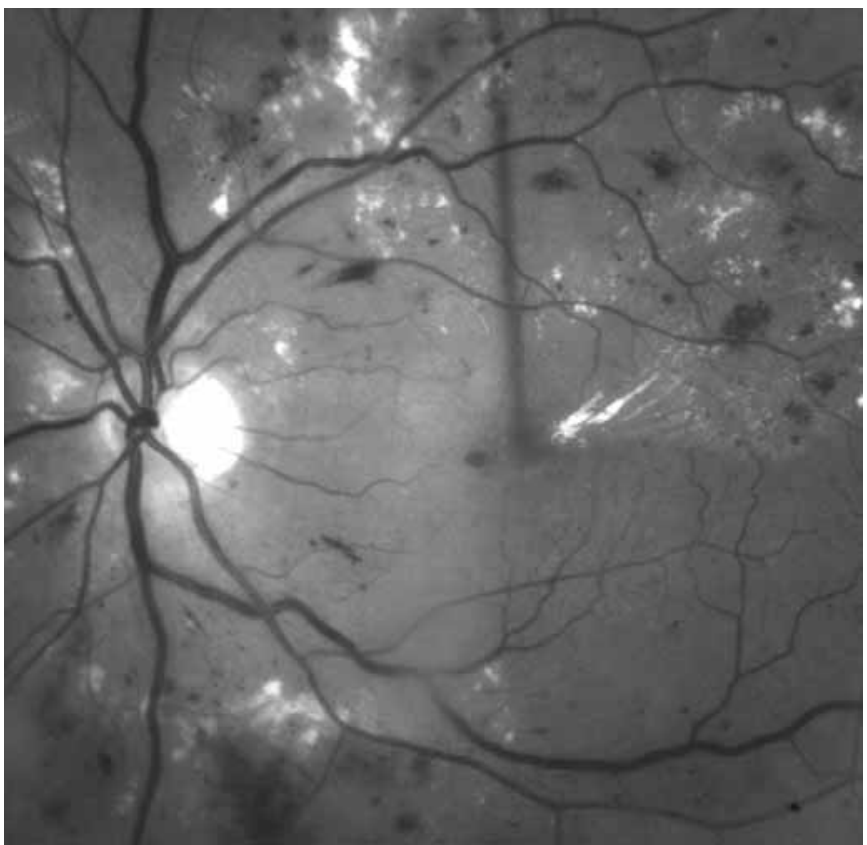

Fig. (2). Patient \#2: OS Retinal green filter photograph (A) and venous phase fluorescein angiogram (B) demonstrating diffuse macular edema with circinate and stellate intra-retinal lipid, dot, blot, and striate hemorrhages and diffuse posterior retinal vascular leakage.

A

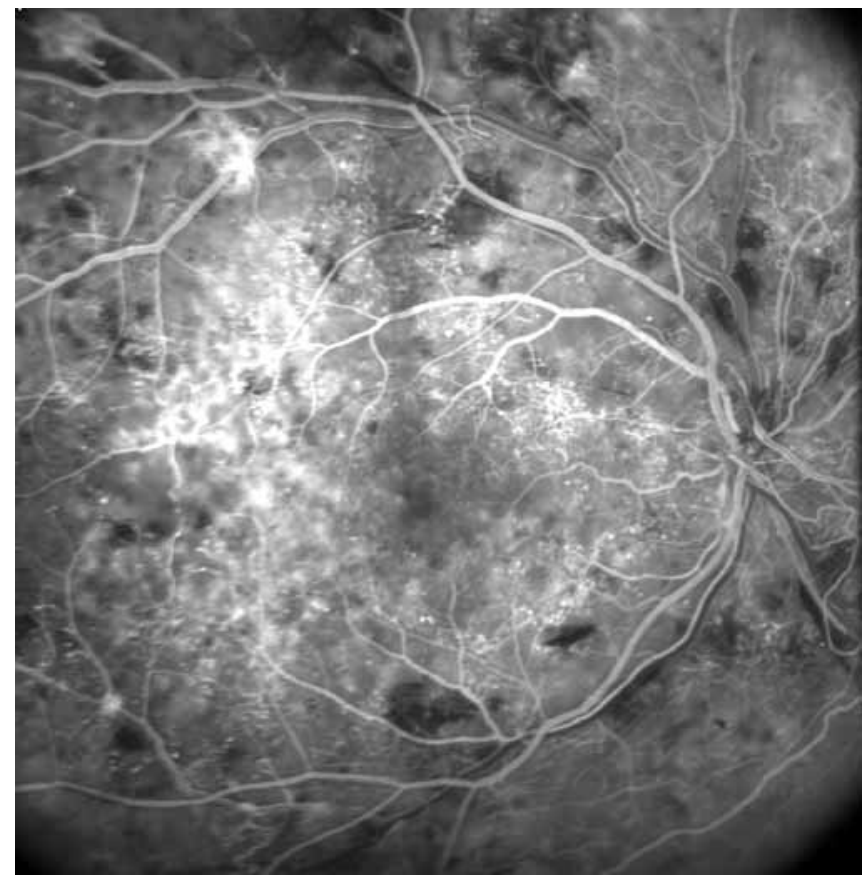

B

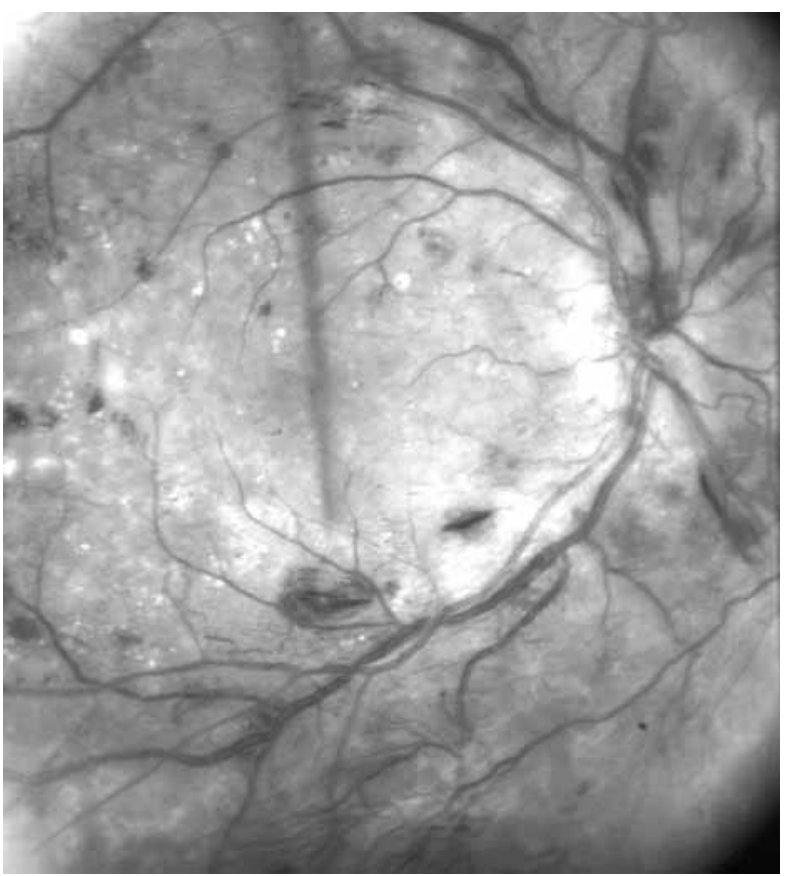

Fig. (3). Patient \#3: OD Retinal green filter photograph (A) and capillary phase fluorescein angiogram (B) demonstrating diffuse retinal hemorrhage and edema with striate hemorrhage, multiple foci of epi papillary and epiretinal neovascularization with diffuse retinal telangiectasia leakage and severe posterior and peripheral capillary non-perfusion.

tion, the edema did not improve and in most cases manifested progressive development of more areas of microvascular leakage as telangiectasia developed around areas of evolving nerve fiber layer infarcts. This was in contrast to the 9 patients without sleep apnea, in which the macular edema in 8 resolved with grid laser and in most cases with one treatment. Ten of the eyes of patients with sleep apnea had undergone panretinal photocoagulation for epi-papillary or epi-retinal neovascularization. In these eyes the neovascularization remained "active" after the scatter photocoagulation and was accompanied by new nerve-fiber-layer infarcts, multiple foci of IRMA and venous dilation or venous beading that persisted after the pan-retinal laser. Seven of the patients had undergone pars plana vitrectomy for complica- 
Table 2. Sleep Characteristics of Apneic Patients

\begin{tabular}{|c|c|c|c|c|c|}
\hline & Median & Mean & Std Dev & Min & Max \\
\hline Hemoglobin $\mathrm{O}_{2}$ sat. Awake (\%) & 97.0 & 96.3 & 1.7 & 92.0 & 98.0 \\
\hline Apneic/hypopneic Events per hour during REM sleep & 57.8 & 55.7 & 17.7 & 25.0 & 89.0 \\
\hline Average duration of events (secs) & 22.4 & 23.3 & 5.9 & 13.7 & 38.0 \\
\hline Total duration of events (minutes) & 74.4 & 82.8 & 51.5 & 9.0 & 162.3 \\
\hline Minimum Hemoglobin $\mathrm{O}_{2}$ sat. during apneic/hypopneic events & 72.0 & 73.5 & 9.5 & 51.0 & 88.0 \\
\hline
\end{tabular}

tions of the neovascularization. Among those without apnea, one eye had undergone scatter photocoagulation with resolution of the epi-retinal neovascularization.

Among the 22 patients identified with an Epworth score of greater than 10 (daytime somnolence very suggestive of sleep apnea), 16 were classified as demonstrating severe obstructive sleep apnea on baseline sleep studies (more than 25 apneic or hypopneic events per hour or producing oxyhemoglobin saturations less than $80 \%$ ) and the other 6 were classified as moderate (10-25 apnea/hypopnea events per hour with no $\mathrm{SaO}_{2}$ below $80 \%$ ). The results of the diagnostic sleep study are presented in Table 2. An average of $44.6 \pm 21.9$ apneic or hypopneic events were recorded per hour, increasing to $55.7 \pm 15.7$ events per hour during REM sleep. The events on average lasted $23.3 \pm 5.9$ seconds with the longest events during the night's recording on average lasting more than a minute. The lowest oxyhemoglobin saturation recorded during the night polysomnogram averaged $73.5 \pm 9.5 \%$ with some recorded as low as $50-60 \%$. The average duration of the apneic or hypopneic events together to- taled $82.8 \pm 51.5$ minutes or approximately one quarter of the time asleep.

All patients underwent blood testing for $\mathrm{HgA} 1 \mathrm{C}, \mathrm{CBC}$, lipid profile, erythrocyte sedimentation rate, $\mathrm{C}$ reactive protein, BUN, and creatinine, and 24 hour urine collection for creatinine clearance and proteinuria. The results are presented in Table 3 for the apneic and non-apneic groups. No statistically significant differences were noted between the groups in the number of patients with abnormalities or in the mean of each abnormality.

\section{DISCUSSION}

This is the first description of a putative association between obstructive sleep apnea syndrome in obese diabetics and an apparent aggravation of their retinopathy. A group of obese diabetic patients with symptoms and polysomnographic evidence of moderate or severe sleep apnea was compared with a cohort that was matched for similar obesity, age, and duration of diabetes but without symptoms suggesting sleep apnea. All of the diabetic patients in the cohort

Table 3. Hematologic and Urine Evaluations of Patients Studied

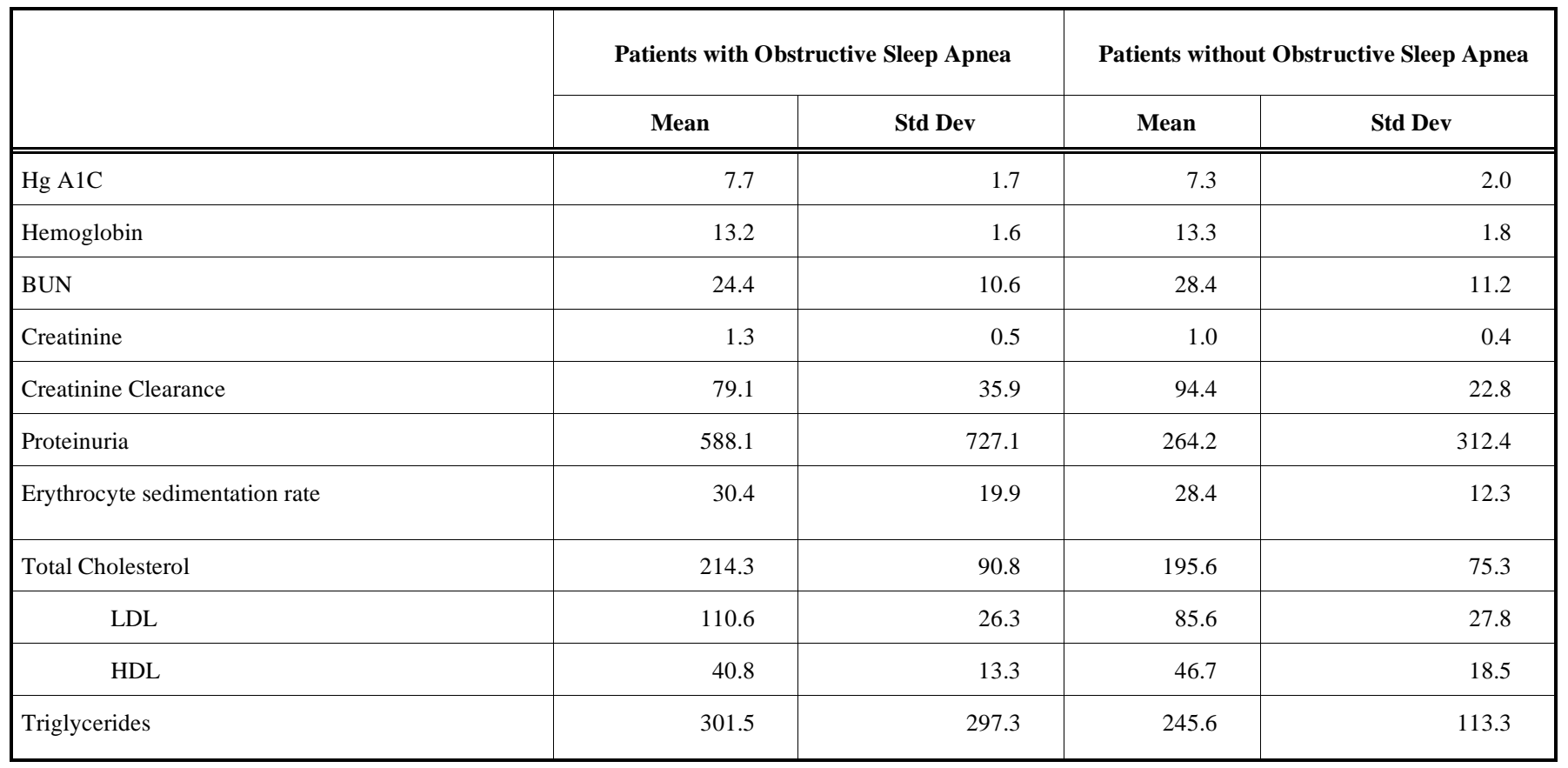


with apnea demonstrated no hypoxemia while awake, but suffered nocturnal oxygen desaturation in the low 70's (and in several patients into the 50's or 60's) secondary to moderate or severe sleep apnea averaging more than 44 episodes per hour. We believe that the repetitive hypoxemia associated with the apneic or hypopneic episodes contributes to the diabetic microvascular injury, producing progressive increase in permeability mixed with ischemia. In these patients the retinopathy characteristically demonstrated multiple nerve fiber layer infarcts with recurring new lesions and was often associated with diffuse, progressive macular edema that was resistant to macular grid laser photocoagulation. In those patients without apnea the retinopathy demonstrated few nerve-fiber layer infarcts and with less peripheral ischemia. The edema that was observed was due to fewer and more localized areas of leakage and in all but one, resolved with laser photocoagulation.

In the diabetic retina, because of the reduced capability for an autoregulatory response to oxygen changes [17], the recurrent episodes of severe hypoxemia occurring with the apneic events (equivalent to sleeping one-quarter of the night on the top of Mt. Everest), are thought to have the potential to injure the retinal microvasculature aggravating the diabetic microvascular hyper-permeability.

Although blood pressures were not measured during the sleep studies in these patients, acute rapid elevations of 10$30 \mathrm{mmHg}$ or more of pulse pressure and mean systemic pressure have been previously reported toward the end of each apneic or hypopneic episode and with a gradual, progressive, elevation of $20-30 \mathrm{mmHg}$ in baseline blood pressure observed through the night $[4,5]$ The recurrent rapid increases in pulse pressure and mean pressure, occurring toward the end of each hypopneic or apneic episode, as well as the progressive elevation of baseline blood pressure through the night, we speculate, might result in an exaggerated effect on the microvasculature because of the concurrent hypoxemia. An increased prevalence of chronic hypertension during the day is reported with sleep apnea [24, 25], and treatment of the obstructive sleep apnea often improves the daytime hypertension control [26]. Chronic hypertension has been reported to be associated in diabetics with increased incidence of nerve-fiber-layer infarcts, pre-proliferative retinopathy and macular edema [2, 27, 28]. Nineteen of the 22 diabetic patients with apnea in this study were noted to have a history of hypertension at the time of diagnosis of obstructive sleep apnea, and of these all of the patients were on two or more medications. However, in this study, among the nonapneic cohort, although blood pressures were similar, the retinal manifestations of nerve-fiber-layer infarcts, macular and peripheral capillary non-perfusion, and macular edema were significantly different.

Although not evaluated in this group of diabetics, obese patients with severe obstructive sleep apnea often suffer hypercapnea that accompanies the hypoxemia during each apneic episode [14]. In the retina, hypercapnea produces minimal vessel dilation (Sinclair, unpublished data) but has been demonstrated to cause cerebrovascular dilation and elevated CSF pressure in patients with sleep apnea [29]. Recently in non-diabetic patients with obstructive sleep apnea, cases of optic disc edema have been reported with elevated CSF pressure [30]. Elevated CSF pressure would increase the retinal venous outflow pressure and would result in elevated retinal capillary hydrostatic pressure, further exacerbating the macular edema from the hyper-permeable capillaries observed in diabetic retinopathy. In 6 of the patients with obstructive sleep apnea, disc edema was noted on fundus photography and with what was thought to be exceptional disc leakage on fluorescein angiography, although in all of the cases with apnea there was some degree of disc leakage associated with the diabetic microangiopathy. Capillary leakage on the surface of the disc, however, may represent a manifestation of the hyper-permeability observed with wide-spread diabetic microangiopathy, ischemia and the liberation of vascular endothelial growth factor, a permeability enhancer [31]. In addition, there may be some exacerbation induced by hypercapnea and the CNS effects, but to what degree, we cannot ascertain. Twenty of the 22 patients with sleep apnea were noted to have macular edema and all reported a significant deterioration in their vision in the morning on awakening compared with 5 of the 9 with macular edema but no sleep apnea. We presume the deterioration in vision to be due to an aggravation of the macular edema at night perhaps by the hemodynamic factors occurring with OSAS that clear on arising from sleep in the morning, but whether hypercapnea occurs and exacerbates the edema must remain as speculative. It is interesting that in the cases of disc edema reported by Purvin [30], there were also vision defects, presumed due to anterior optic nerve ischemia [32], and cases of anterior ischemic optic neuropathy have been reported in patients with obstructive sleep apnea [33]. Among the diabetic patients described here, two presented initially with nonarteritic anterior ischemic optic neuropathy. Djovkar et al. [34] also showed influence of intermittent hypoxia on intravenous glucose tolerance and insulin sensitivity in anaesthetized normal rats.

Other systemic diseases have been reported to be associated with an aggravation of nerve-fiber-layer infarcts and macular edema in diabetics, including poorly controlled diabetes [35], hypertension [20, 21], hyperlipidemia [36-38], anemia $[39,40]$, vasculitis $[36,41]$, and nephropathy [36, 42]. Table 3 presents the evaluation of each of these systemic diseases in the apneic and non-apneic cohorts. Among both cohorts, typical of patients with long duration diabetes and retinopathy, many of the patients demonstrated accompanying abnormalities. There was no significant difference noted between the groups and in most of the cases in both groups, the systemic abnormalities were felt to be under reasonable control with medication. While in occasional patients in both groups, there were significant abnormalities noted, the occurrence was sporadic and was not associated with the worst cases of retinopathy.

We attempted to determine if there were a correlation between each of the measured systemic indices and the severity of the retinopathy (e.g. the number of nerve fiber layer infarcts or the graded severity or area of capillary nonperfusion observed on fluorescein angiography) utilizing the Wilcox sum rank test, but no significant correlations were demonstrated. We also attempted to determine if there was a correlation between the retinopathy and the severity of obstructive sleep apnea, as evaluated by the severest degree of hypoxemia, or the apnea index, (the total number of hypopnea/apnea events per hour). However, no correlation could be determined with confidence. The failure to find a 
significant correlation may be because of the difficulty to determine an adequate method to grade the retinopathy, perhaps because of the great variability in duration of the diabetes and retinopathy or because of the lack of adequate methods to evaluate the severity and duration of hypoxemia, hypertension, or hypercapnea, occurring with OSAS syndrome that are thought to accelerate the retinopathy.

If the OSAS were a major factor contributing to the retinopathy observed in these patients, then adequate treatment should improve the maculopathy or reduce the progressive capillary occlusion caused by repetitive "showers" of nervefiber-layer infarcts that were observed in these patients. Although we recognize that once significant retinal capillary occlusion has developed, it is not reversible and ultimately leads to neovascular proliferation. Sleeping with a nasal mask and apparatus that delivers continuous positive airway pressure (CPAP) or biphasic positive airway pressure (BI$\mathrm{PAP}$ ) is the most common method utilized to treat OSAS and the method utilized in all but four of the patients reported in this series (two had uvulopalatopharyngoplasty and two refused to wear a mask). We have followed 13 of the 22 apneic patients for more than 9 months of continuous nasal mask treatment. In 11 of the 13, the showers of nerve fiber layer infarcts ceased and in the other 2 they were reduced. In 8 patients, the maculopathy (macular edema) regressed spontaneously or improved with laser treatment and has remained stable as opposed to the prior history of progression. In 5 patients the maculopathy (edema mixed with ischemia) has persisted. In 5 patients who had either refused treatment or who had significant compliance problems (all of whom were diagnosed with severe obstructive sleep apnea), one patient died of an MI during the night, and the other 4 have continued to manifest severe macular edema with on-going nervefiber-layer infarction, and one developing proliferative retinopathy. We recognize, however, that measurements of patient compliance with using the CPAP or BIPAP apparatus as well as its efficacy in reversing the hypoxemia, hypercap- nea and hypertension are difficult to assess. In a recent study of 32 patients treated with nasal CPAP, half the subjects consistently used the device over the nine-week trial while the other half had a wide range of daily use [43]. Other studies have also reported similar rates of poor compliance [44, 45]. Surgical treatment or tongue and mandible repositioners are less commonly employed, but appear to have limited success in the obese population, and are equally difficult to evaluate. Most often successful treatment is defined by the subjective improvement in daytime somnolence which may be related to improvement in arousals due to snoring but which may not reflect an improvement in the severity or frequency of the hemodynamic effects of the apneic episodes.

In conclusion, while this paper presents an initial descriptive association of obstructive sleep apnea with an accelerated course of established ischemic and edematous diabetic retinopathy, we believe there is sufficient evidence to pursue further evaluation of the effect of OSAS and its treatment on patients with earlier stages of diabetic retinopathy. In this pilot series, sequential patients with significant somnolence, as evidenced by a high Epworth score that has been highly associated with the OSAS syndrome [46] were compared with a retrospectively selected group of patients with low Epworth scores that correlate with low rates of OSAS, but the latter patients were not tested with polysomnography. Patients with obstructive sleep apnea should be evaluated against a cohort of those without in a prospective fashion in which blood pressure, oxyhemoglobin saturation, and pC02 are measured in a baseline study and then in those with the OSAS syndrome, during a CPAP/BIPAP titration protocol, and then both cohorts of patients need to be followed, with the apneic cohort using a nasal mask that is designed to evaluate compliance. Only in this fashion can it be determined if this syndrome, which has been associated with nocturnal myocardial and cerebrovascular infarction, indeed has deleterious effects on diabetic retinopathy, and whether treatment reduces or halts those effects. Perhaps through

\section{Table 4. The Epworth Sleepiness Scale [46]}

How likely are you to doze off or fall asleep in the following situations, in contrast to feeling just tired? Use the following scale to choose the most appropriate number for each situation.

$0=$ would never doze

$1=$ slight chance of dozing

$2=$ moderate chance of dozing

$3=$ high chance of dozing

\begin{tabular}{|l|l|}
\hline Situation & Chance of Dozing \\
\hline \hline Sitting and reading & \\
Watching TV & \\
Sitting, inactive in a public place (e.g. a theater or a meeting) & - \\
As a passenger in a car for an hour without a break & - \\
Lying down to rest in the afternoon when circumstances permit & - \\
Sitting and talking to someone & - \\
Sitting quietly after lunch without alcohol & - \\
In a car, while stopped a few minutes in traffic
\end{tabular}

Comment: The test requires that the patient estimate his behavior in various situations some of which would produce dozing in most people. A score of 10 or greater is considered positive for pathological sleepiness (In original validation, normal controls scored 5.9 \pm 2.2 while patients with obstructive sleep apnea scored 11.7 \pm 4.6 ) 
those studies as well as through studies on the prevalence of OSA syndrome in obese diabetics, light will be shed on its influence of diabetic microangiopathy, not only in the retina, but also on the renal, cerebral, and myocardial microvasculature. These observations also may shed light on the causes of the high prevalence of vitreous hemorrhage occurring with proliferative diabetic retinopathy at night and on the aggravation of vision and macular edema observed on awakening.

In the interim, because of the high prevalence of obstructive sleep apnea in obese patients, we believe it is prudent for physicians to evaluate obese diabetics for this syndrome, especially if they snore, have fragmented sleep patterns, or report daytime somnolence (Epworth score 10 or greater, please see Table 4) [46]. It must also be remembered that a substantial percentage of OSAS patients do not complain of excessive daytime somnolence. The epidemic of diabetes associated with obesity occurring in the United States, we believe, will likely result in an explosion of aggressive retinopathy with vision morbidity if the obstructive sleep apnea that is commonly associated with obesity is not recognized and aggressively treated. In addition, though obesity is one of the greatest risk factors for obstructive sleep apnea, the problem still occurs at significant rates of $10-30 \%$ among individuals close to ideal body weight $[25,47]$. We would recommend evaluation of diabetics who have the above symptoms with chronic hypertension or who manifest patterns or accelerated course of retinopathy similar to the patients described in this paper.

\section{REFERENCES}

[1] The International Classification of Sleep Disorders Diagnostic and Coding Manual, Allen Press (1990).

[2] Zwillich CT, Devlin T, White D, et al. Bradycardia during sleep apnea: characteristics and mechanisms. J Clin Invest 1982; 69: 1286-92.

[3] Guilleminault C, Connolly S, Winkle R, et al. Cyclic variations in heart rate in sleep apnea syndrome: mechanism and usefulness of $24 \mathrm{~h}$ electrocardiography as a screening technique. Lancet 1984; 1 : 126-31.

[4] Tilkian AG, Guilleminault C, Schroeder JS, et al. Hemodynamics in sleep induced apnea. Ann Intern Med 1976; 85: 714-9.

[5] Stoohs R, Guilleminault C. Cardiovascular changes associated with obstructive sleep apnea. J Appl Physiol 1992; 72: 582-9.

[6] Hla K, Young T, Bidwell T, et al. Sleep apnea and hypertension: a population based study. Ann Int Med 1994; 120: 382-8.

[7] Chaouat A, Weitzenblum E, Krieger J, et al. Pulmonary hemodynamics in the obstructive sleep apnea syndrome: results in 220 consecutive patients. Chest 1996; 109: 380-6.

[8] McNamara SG, Cistulli PA, Strohl KP, et al. Clinical aspects of sleep apnea. In: Sullivan C, Saunders NA, Eds. Sleep and breathing, New York: Marcel Dekker 1993; vol. 14: pp. 493-528.

[9] Sajkov D, Cowie RJ, Thornton AT, et al. Pulmonary hypertension and hypoxemia in obstructive sleep apnea syndrome. Am J Respir Crit Care Med 1994; 149: 416-22.

[10] Dyken ME, Somers VK, Yamada T, et al. Investigating the relationship between stroke and obstructive sleep apnea. Stroke 1996; 27: 401-7.

[11] Kuhlmann U, Becker HF, Birkhahn M, et al. Sleep apnea in patients with end-stage renal disease and objective results. Clin Nephrol 2000; 53: 460-6.

[12] Kushida CA, Efron B, Guilleminault C. A predictive morphometric model for obstructive sleep apnea syndrome. Ann Intern Med 1997; 127: $581-7$.

[13] Shelton KE, Woodson H, Gay S, et al. Phayrngeal fat in obstructive sleep apnea. Am Rev Respir Dis 1993; 148: 462-6.

[14] Weiss J, Launois S, Anand A. Cardiorespiratory Changes in SleepDisordered Breathing. In: Kryger, Roth, Dement, Eds. Principles and Practice of Sleep Medicine, Philadelphia: W.B. Saunders Company, 2000; pp. 859-68.
[15] Mokhad AH, Ford ES, Bowman BA, et al. Diabetes trends in the U.S.: 1990-1998. Diabetes Care 2000; 23: 1278-83.

[16] Bresnick GW, de Venecia C, Myers FL, et al. Retinal ischemia in diabetic retinopathy. Arch Ophthalmol 1975; 93: 1300.

[17] Sinclair SH, Grunwald JE, Riva CE, et al. Retinal vascular autoregulation in diabetes mellitus. Ophthalmology 1982; 98: 74850 .

[18] Grunwald JE, Riva CE, Brucker AJ, et al. Altered retinal vascular response to $100 \%$ oxygen breathing in diabetes mellitus. Ophthalmology 1984; 91 : 1447.

[19] Grunwald JE, Brucker AJ, Schwartz SS, et al. Diabetic glycemic control and retinal blood flow. Diabetes 1990; 39: 602-7.

[20] Daniels S, Lipman M, Burke M, et al. The prevalence of retinal vascular abnormalities in children and adolescents with essential hypertension. Am J Ophthalmol 1991; 111: 205-8.

[21] Viberti GC, Messent J. Hypertension and diabetes: critical combination for micro-and macrovascular disease. Diabetes Care 1991; 14: 4-7.

[22] Knowler WC, Bennett PH, Ballintine EJ. Increased incidence of retinopathy in diabetics with elevated blood pressure. $\mathrm{N}$ Engl J Med 1980; 302: 645-50.

[23] Lugaresi E, Cirignotta F, Gerardi R, et al. Snoring and sleep apnea: Natural history of heavy snorers disease, Chapter 4. In: Guillenminault C, Partinen M, Eds. Obstructive Sleep Apnea Syndrome: Clinical Research and Treatment, New York: Raven Press, Ltd, 1990; pp. 25-36.

[24] Carlson JT, Hedner JA, Eijell H, et al. High prevalence of hypertension in sleep apnea patients independent of obesity. Am J Respir Crit Care Med 1994; 150: 72-7.

[25] Young T, Palta M, Dempsy J, et al. The occurance of sleepdisordered breathing in middle-aged adults. N Engl J Med 1993; 328: 1230 .

[26] Mayer J, Becker H, Brandenberg U, et al. Blood pressure and sleep apnea: results of long term nasal continuous positive airway pressure therapy. Cardiology 1991; 79: 84-92.

[27] Fuller JH, Stevens LK. Epidemiology of hypertension in diabetic patients and implications for treatment. Diabetes Care 1991; 14: 812.

[28] Kearns M, Hamilton AM, Kohner EM. Excessive permeability in diabetic maculopathy. Br J Ophthalmol 1979; 63: 489-97.

[29] Sugita Y, Lijima S, Teshima Y, et al. Marked episodic elevation of cerebrospinal fluid pressure during nocturnal sleep in patients with sleep apnea hypersomnia syndrome. Electroencephalogr Clin Neurophysiol 1985; 60: 214-9.

[30] Purvin VA, Kawasaki A, Yee RD. Papilledema and obstructive sleep apnea syndrome. Arch Ophthalmol 2000; 118: 1626-30.

[31] Hovind P, Tarnow L, Oestergaard PB, et al. Elevated vascular endothelial growth factor in type 1 diabetic patients with diabetic nephropathy. Kidney Int Suppl 2000; 75: S56-61.

[32] Lee AG. Three questions on the role of sleep apnea syndrome in optic disc edema. Arch Ophthalmol 2001; 119: 1225.

[33] Mojon DS, Mathis J, Zulauf M, et al. Optic neuropathy associated with sleep apnea syndrome. Ophthalmology 1998; 105: 874-7.

[34] Djovkar A. Influence of intermittent hypoxia on intravenous glucose tolerance and insulin sensitivity in anaesthetized normal rats. Horm Metab Res 1983; 15: 254-5.

[35] Diabetes Control and Complications Trial Research Group, Diabetes control and complications trial (dcct): update. Diabetes Care 1990; 13: 427-33.

[36] Brown GC, Brown MM, Hiller T, et al. Cotton-wool spots. Retina 1985; 5: 206-14.

[37] Larsson LI, Alm A, Lithner F, et al. The association of hyperlipidemia with retinopathy in diabetic patients aged 15-50 years in the county of Umea. Acta Ophthalmol Scand 1999; 77: 585-91.

[38] Su DH, Yeo KT. Diabetic retinopathy and serum lipids. Singapore Med J 2000; 41: 295-7.

[39] Bosman DR, Winkler AS, Marsden JT, et al. Anemia with erythropoietin deficiency occurs early in diabetic nephropathy. Diabetes Care 2001; 24: 495-9.

[40] Merin S, Freund M. Retinopathy in severe anemia. Am J Ophthalmol 1968; 66: 1102-6.

[41] Duker JS, Brown GC, McNamara JA. Proliferative sarcoid retinopathy. Ophthalmology 1988; 95: 1680-7.

[42] Zander E, Herfurth S, Bohl B, et al. Maculopathy in patients with diabetes mellitus type 1 and type 2: associations with risk factors. Br J Ophthalmol 2000; 84: 871-6. 
[43] Weaver TE, Kribbs NB, Pack AL, et al. Night to night variability in CPAP use over the first three months of treatment. Sleep 1997; 20: $278-83$.

[44] Engleman HM, Martin SE, Douglas NJ. Compliance with CPAP therapy in patients with sleep apnea/hypopnea syndrome. Thorax 1994; 49: 263-6.
[45] Meurice JC, Dore P, Paquereau J, et al. Predictive factors of long term compliance with nasal continous positive airway pressure treatment in sleep apnea syndrome. Chest 1994; 105: 429-33.

[46] Johns MW. A new method for measuring daytime sleepiness: The epworth sleepiness scale. Sleep 1991; 14: 540-5.

[47] Guilleminault C, Tilkian A, Demant WC. The sleep apnea syndromes. Annu Rev Med 1976; 27: 465-84.

(C) Unver et al.; Licensee Bentham Open.

This is an open access article licensed under the terms of the Creative Commons Attribution Non-Commercial License (http://creativecommons.org/licenses/by-nc/3.0/) which permits unrestricted, non-commercial use, distribution and reproduction in any medium, provided the work is properly cited. 